\title{
«Die Medizin wird immer mehr zur Molekularmedizin»
}

\section{Interview: Matthias Scholer ${ }^{c}$}

a Ph.D. Life Sciences, Projektleiterin Klinische Bioinformatik, SIB Schweizerisches Institut für Bioinformatik;

${ }^{b}$ Ph.D. Biotechnologie, Senior Director Contract R\&D Unit, Universitätsspital Basel; ${ }^{c}$ Online- und Printredaktor SÄZ

Heutzutage gehören mehrköpfige, interdisziplinäre Boards in molekularer Onkologie zum Klinikalltag grösserer Institutionen. Doch auch auf Stufe Grundversorgung werden im Zuge der Personalisierten Medizin künftig neue Herausforderungen auf die Hausärztinnen und Hausärzte zukommen. Auch sie brauchen ein breites Wissen rund um pathologische Veränderungen auf Genebene und die vorhandenen, molekularen Therapiemöglichkeiten.

\begin{abstract}
Insbesondere in der Onkologie gewinnt das molekulare Profiling einer Gewebeprobe immer mehr an Bedeutung. Für Ärztinnen und Ärzte, die in ihrem Alltag nicht regelmässig mit solchen Technologien zu tun haben, ist dieses Thema häufig eine Blackbox, von der sie sich ausgeschlossen fühlen. Mit Recht? Luca Quagliata (LQ): Nein, definitiv nicht. Alle können und sollten den Prozess der Genomsequenzierung in der Theorie verstehen lernen. Dieses Wissen bildet die Basis, um im Praxisalltag die richtigen Schlüsse aus den Resultaten eines pathologischen Berichts ziehen zu können und in eine optimale Therapie einfliessen zu lassen.
\end{abstract}

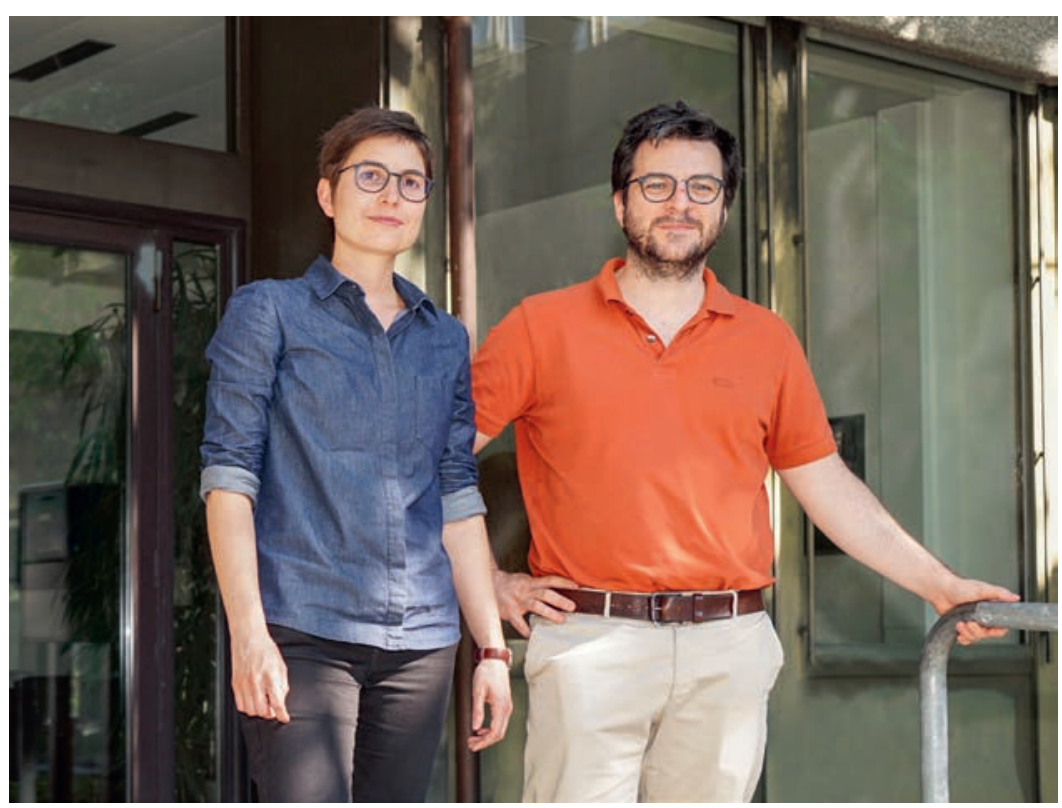

Aitana Lebrand und Luca Quagliata engagieren sich für die Wissensvermittlung im Bereich der Molekularmedizin.
Aitana Lebrand (AL): Deshalb ist auch eines der Ziele, die wir im Rahmen einer schweizerischen Arbeitsgruppe verfolgen, die Standardisierung der molekularpathologischen Berichte. Wir wollen keine zwanzigseitigen Berichte, sondern solche, die einfach lesbar und allgemein verständlich sind. Trotzdem braucht es einen Grundstock an molekularmedizinischem Wissen, um die Informationen optimal nutzen zu können.

Welche Rolle werden die Grundversorger bei Patienten, bei denen eine gezielte Krebstherapie zur Anwendung kommt, übernehmen?

LQ: Die Bedeutung ihrer Rolle als gatekeepers wird zunehmen. Diese beginnt einerseits mit der detaillierten Informationszusammenstellung im Rahmen der Anamneseaufnahme. Schliesslich werden die meisten genetischen Veränderungen durch familiäre oder externe Faktoren wie das Arbeitsumfeld oder den Lebensstil, dazu gehört zum Beispiel das Rauchen, verursacht. Folglich sind die Informationen über einen Patienten für eine Diagnosestellung und optimale Therapiewahl von zentraler Bedeutung. Andererseits wird das Monitoring der Patienten nach einer personalisierten Krebstherapie vermehrt die Aufgabe der Hausärztinnen und Hausärzte. Da die Überlebensrate dank diesen neuen Therapien in vielen Fällen zunimmt, wird die Verbindung zwischen Hausarztpraxis und Patient in Zukunft tendentiell zunehmen. Deshalb ist es auch wichtig, dass Hausärzte wissen, welche Medikamente im Bereich der personalisierten Krebstherapie auf dem Markt sind, wie sie funktionieren und worauf sie beim Monitoring der Patienten achten müssen. 


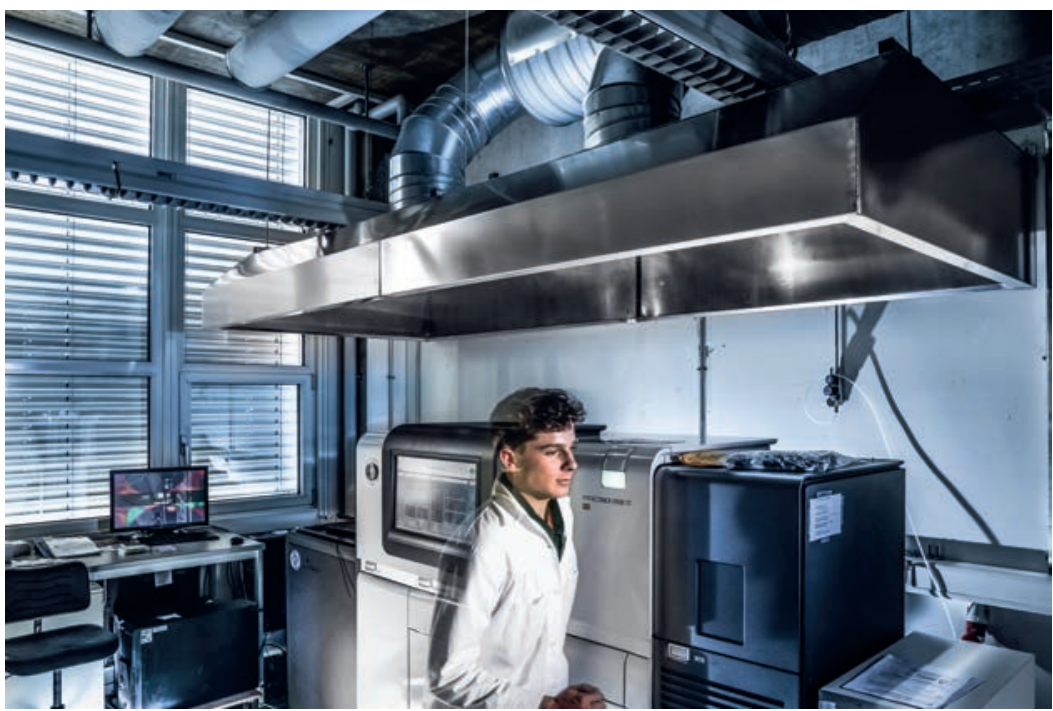

Gensequenzierung ist heute bereits fester Bestandteil der medizinischen Diagnostik.

\section{Wie können sich Ärztinnen und Ärzte dieses Wissen aneignen?}

$A L$ : Unsere Schweizer Arbeitsgruppe ist eine ideale Plattform, um die klinischen Bedürfnisse im Bereich der personalisierten Onkologie zu verstehen und entsprechende Weiterbildungsmöglichkeiten anzubieten. Aus diesen Gesprächen heraus startet SIB in diesem Herbst gemeinsam mit den Universitätsspitälern Basel und Lausanne ein CAS in Personalisierter Molekularer Onkologie.

\section{Ein Kurs ausschliesslich für Onkologen also?}

$A L$ : Nein. Der Kurs richtet sich an alle in eine Diagnosestellung und Therapieentscheide involvierten Fachpersonen - vom Allgemeinmediziner bis zum Onkologen, Pathologen, Bioinformatiker und Genetiker. Das Ziel des Zertifikatkurses ist es, die Technologien, Prozesse, Möglichkeiten, aber auch Limitationen der Molekularpathologie und der personalisierten Therapien im Detail aufzuzeigen. So weiss jede beteiligte Person, was für welchen Schritt in der Pipeline wichtig ist und wie sie mit ihrer eigenen Arbeit zum bestmöglichen Output beitragen kann.

\section{Werfen wir einen Blick auf die stationären Institu- tionen und die Forschung. Welche Aktivitäten stehen in diesem Bereich an?}

$A L$ : Das mittelfristige Ziel ist die Harmonisierung und Standardisierung der molekularbiologischen Prozesse, aber auch der Interpretation der Laborresultate. Es gibt zahlreiche Genvarianten, bei denen wir uns nicht sicher sind, wie pathogen sie sind. Somit interpretiert jedes Spital diese unterschiedlich. Es ist je- doch im Interesse aller, dass eine Mutation schweizweit standardisiert interpretiert wird. $\mathrm{Zu}$ diesem Zweck haben sich die Universitätskliniken und forschungskompetente Spitäler unter der Führung des SIB und der Schweizer Arbeitsgruppe zusammengeschlossen, um in den kommenden drei Jahren eine Datenbank aufzubauen, welche garantiert, dass alle beteiligten Partner auf qualitativ hochstehende Daten zugreifen und damit arbeiten können. Dieses Projekt wurde seither im Rahmen des Swiss Personalized Health Network genehmigt.

$L Q$ : Basierend auf diesen Daten kann dann ein Expertenpanel für jede Genvariante einen Konsens über deren Pathogenität finden. Da in der heutigen Diagnostik und während des Prozesses der Therapiefindung multidisziplinäre Teams zusammenarbeiten, ist es von zentraler Bedeutung, dass alle Beteiligten ein Grundwissen in Molekularmedizin haben. Nur so können die Teams eine gemeinsame Sprache finden.

\section{Eine Tendenz, die sich auf verschiedene Gebiete der Medizin übertragen lässt, oder?}

$A L$ : Absolut. Denken wir nur an die medizinische Genetik bei Neugeborenen oder im Bereich der Infektiologie. Es ist zudem davon auszugehen, dass künftig auf Stufe Hausarzt mit vermehrten Nachfragen bezüglich Gentests für Risikofaktoren zu rechnen ist. Die Basis für all diese Einsatzmöglichkeiten ist ein Grundverständnis für Methoden wie die Genomsequenzierung. Denn die Medizin wird immer mehr zur Molekularmedizin.

\section{CAS in Personalisierter Molekularer Onkologie}

Als erstes seiner Art in der Schweiz ist das CAS in Personalisierter Molekularer Onkologie offen für das gesamte Personal im Gesundheitswesen, das sich Wissen über die Methoden zur Generierung, Analyse und Interpretation des molekularen Profils von $\mathrm{Pa}$ tienten aneignen oder sein Wissen erweitern und sich mit den dazugehörigen Herausforderungen auseinandersetzen möchte. Die erste Runde beginnt im November 2018. Einschreibung und detaillierte Informationen: www.pmo.unibas.ch

\section{Bildnachweis}

Porträt: Matthias Scholer

Labor: SIB Nicolas Righetti | Lundi13 